\title{
Conversation with Vittorio Gallese about empathy and aesthetic experience
}

\author{
PERLA GIANNI FALVO, Art Perception International, Italy
}

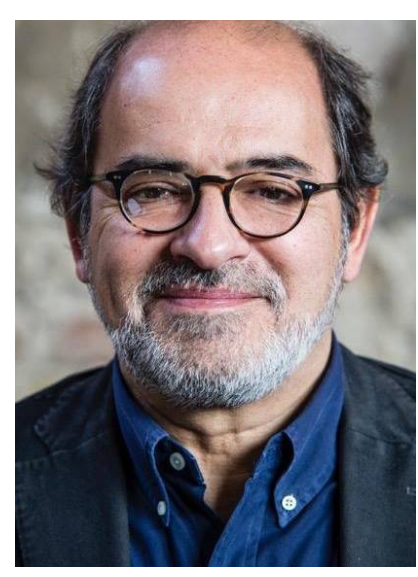

Vittorio Gallese is professor of Psychobiology at the University of Parma, Italy, and was professor in Experimental Aesthetics at the University of London, UK (20162018). He is an expert in neurophysiology, cognitive neuroscience, social neuroscience, and philosophy of mind. Gallese is one of the discoverers of mirror neurons. Gallese has been doing research at the University of Lausanne, Switzerland, at the Nihon University, Tokyo, Japan, at the University of California at Berkeley and at the Berlin School of Mind and Brain of the Humboldt University of Berlin. He has been George Miller visiting professor at the University of California at Berkeley. His research attempts to elucidate the functional organization of brain mechanisms underlying social cognition, including action understanding, empathy, language, mindreading and aesthetic experience.

Key words:

Art perception, Neurosciences, Mirror neurons.

\section{SDH Reference:}

Perla Gianni Falvo. 2018. Conversation with Vittorio Gallese about empathy and aesthetic experience. SDH, 2, 1, XXX-XLVII.

DOI : $10.14434 /$ sdh.v2i1.27926

Perla Gianni Falvo: What do you remember about your last experience in a museum or an art show?

Vittorio Gallese: The last exhibition I saw with some attention is "States of Mind" at the Palazzo dei Diamanti in Ferrara. Among the works on display a painting by Pellizza da Volpedo (Fig. 1a-b) struck me the most. It depicts a seated woman with a slightly bony hand grasping the arm of the chair. To underline how the movement is fundamental, in the Conference "Empathy and Aesthetic Experience" [Gallese 2018a], I have placed her next to some details of the lament on the Dead Christ of Bologna by Niccolò dell'Arca (Fig. 1c-d).

In Pellizza's work there is a movement of the hand that grasps the arm of the chair, which is as expressive of her character as her face. The pose shows an almost resigned abandonment that tells

Author's address: Perla Gianni Falvo, Art Perception International, Via Orcagna 53, 50121, Florence, Italy; email: perla@artperception.org.

(C) [2018] by the author; licensee Studies in Digital Heritage, IU, Bloomington (IN), USA. This article is an open access article distributed under the terms and conditions of the Creative Commons Attribution License (CC BY-NC). 
a lot about this woman, and thanks to our mirror neurons we reproduce her gesture in order to know it ${ }^{1}$.

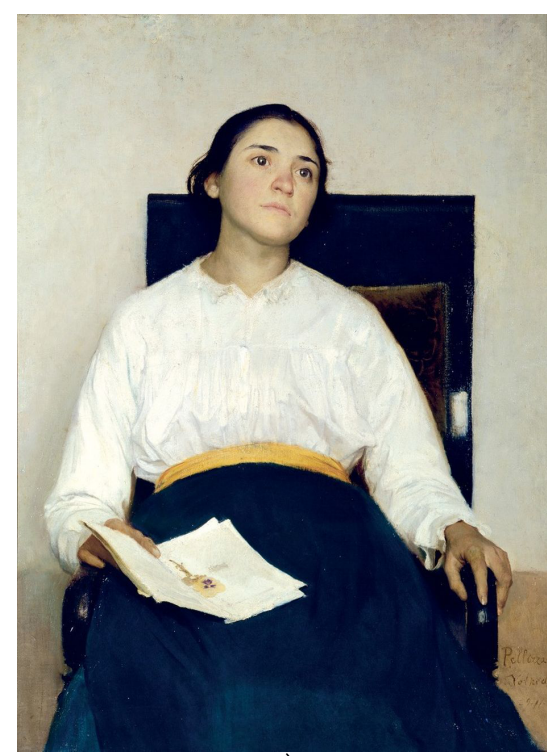

a)

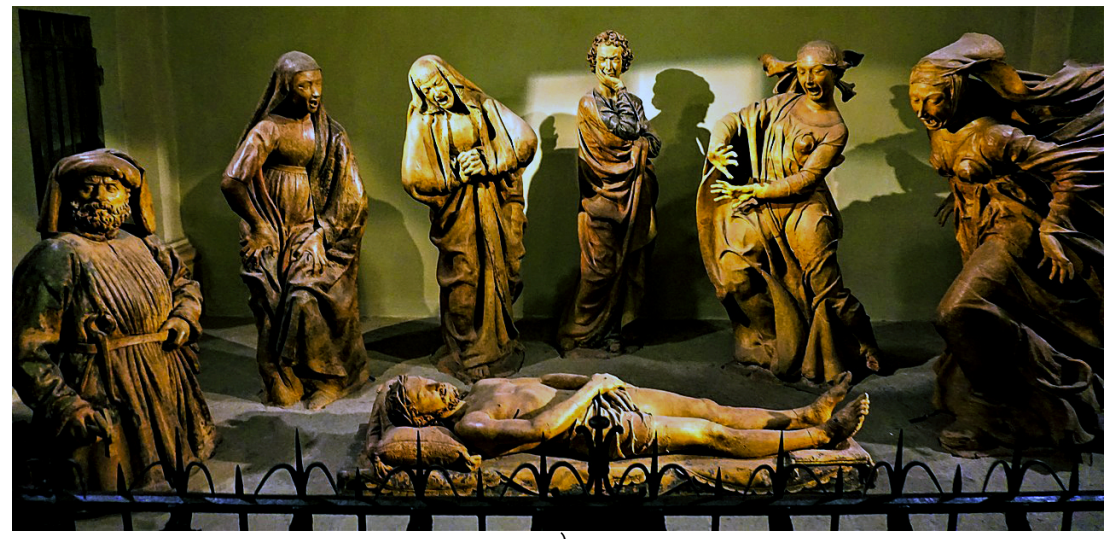

c)

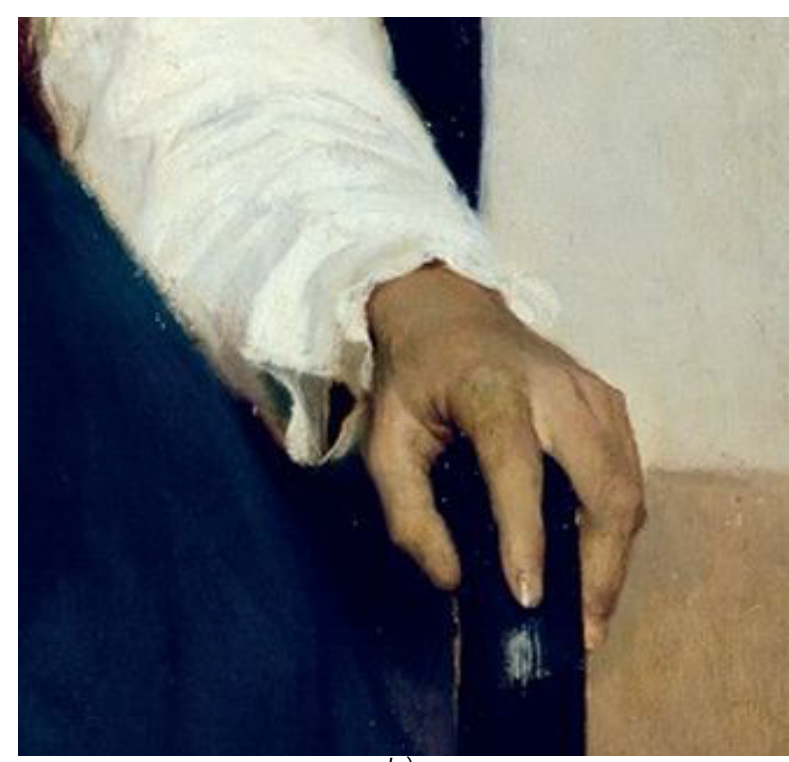

b)

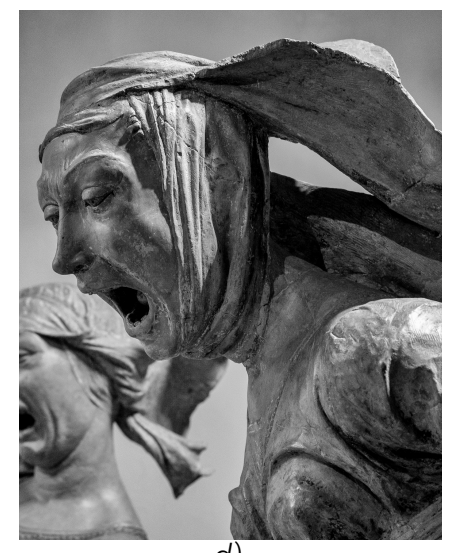

d)

Figure 1. Artistic works activating an empathic reaction of the observer: a) Giuseppe Pellizza da Volpedo, Memory of a pain (portrait of Santina Negri), oil on canvas, 1889 (curtesy of Carrara Academy Foundation); $b$ ) detail of the hand in a); c) Niccolò dell'Arca, Lamentation over the dead Christ, terracotta sculptural group, 1463$1490 ;$ d) the desperate expression of Mary Magdalene in c).

\footnotetext{
${ }^{1}$ Mirror neurons are a class of neurons that are selectively activated both when we perform an action and when we observe it being performed by someone else. The neurons of the observer "mirror" what is taking place in the mind of the person being watched, as if the observer himself were performing the action, the motor areas corresponding to those muscles are activated in our brain [Rizzolati and Sinigaglia 2006].
} 
On the one hand, we simulate the movement, but to the extent that this gesture has its own emotional prosody - which is expressed when the artist is able to make you understand not only what the character portrayed in the sculpture or painting is doing, but also the way he does it, we perceive the affective tonality. We understand if the gesture is abrupt, shy, pensive, kind or aggressive. Today, we know that the brain maps these vital forms or forms of vitality as Daniel Stern ${ }^{2}$ called them, and they activate a particular structure, the insula that acts as a hinge between what happens in the external world and in the internal world. It is an interface between exteroception, that is our ability to represent and experience what happens outside of us, and enteroception, that is what happens simultaneously within us and our body, like the frequency of heart, breath rate, blood pressure, etc.

The key word for me in general is relationship. We are beings that dynamically self-delimit and therefore self-determine, only to the extent that we can relate. Even if our brain is predisposed for language, if we are not exposed to language - that is, to speaking humans- in a well-defined time window, we do not have the capacity to develop this potential. One can cite the example of the feral child, the so-called "child wolf" in whom language does not develop anymore because of isolation from family and society. Therefore, even the skills to which we are naturally predisposed require the presence of and interaction with other individuals.

PGF: We could therefore say that art is nothing but a mediated form of relationship. Where the artwork, whatever its form, becomes a mediator between two subjectivities, that of the author and that of the viewer. In the final analysis, they are always two subjectivities that represent the human being and therefore, in the end, everything returns to one origin, to the way of expressing life.

VG: Yes of course.

PGF: And what's the best thing you expect to happen when you're in front of a work of art?

VG: The visit to a museum, to an exhibition, is almost the celebration of a ritual in which I fully recognize myself, where we find some teasers: the artist's name, the parallel comparison between two artists, the deepening of a particular dimension of an artist , a year of her/his artistic production, etc. Often nothing happens, in the sense that I appreciate, I see, I learn, I know, but the surprise of finding myself somehow in front of something that I had not been able to anticipate, might be missing. An occasion in which this did happen was when I visited one of the most beautiful exhibitions I have seen. It was in 2002 in the New Tate and was an exhibition that proposed the parallel careers of Matisse and Picasso from the point of view of their artistic production, paintings, sculptures, graphic works, in which the mutual influences could be seen very well. Well, in short, almost at the end of the show, I proceeded into yet another room and I can't remember if I was looking for someone I had come with, so I turned around and saw a big white wall with a very colorful picture by Matisse, mostly red that obscures absolutely everything around it. At that point I went back, stopped, looked at it, and then before leaving I went back and looked at it again.

That was an aesthetic experience that left a mark, because this picture and above all the way in which it presented and manifested itself, the scenographic set-up of this white wall with this small picture

\footnotetext{
${ }^{2}$ Daniel N. Stern, psychiatrist (August 16, 1934 - November 12, 2012) in 2010, he published "Forms of Vitality: Exploring Dynamic Experience in Psychology, the Arts, Psychotherapy and Development," which used new understandings of neuroscience to explain human empathy.
} 
(it must have been at most 20 by $30 \mathrm{~cm}$ ) struck me deeply, leaving an indelible mark in my memory. That experience was so powerful that I still talk about it 15-16 years later.

PGF: Of course, the layout directs the relationship. This indicates that when a contact is deep, it becomes indelible, because it marks itself in the history of our life, in the meaning of what we are. We experience a sort of epiphany.

VG: Exactly, "epiphany" is the correct term. These moments of recognition, of epiphany, I can count them on the fingers of one hand. There is another that I remember very well, and I can also tell you precisely when it happened, because it concerns a period of my life of which I can very well reconstruct the timing. I was in Venice for a short vacation back from Japan, where I was working. It was an exhibition of Francis Bacon, a painter I knew, but not very well. In fact, this experience greatly reduced my enthusiasm for another contemporary painter, Lucian Freud. After Bacon you find it hard to go back to Freud, you continue to appreciate him but in short, you put them on two different levels.

The work I think was titled Water Jet (Fig. 2), and it is a white jet of water that comes out of a faucet, with a strength, a power, a dynamism an energy... I really was amazed, and from the moment I saw it I turned back to review all the other Bacon paintings I had just seen. So somehow, it's a prototypical moment. It is a moment that opens up to you some characteristic elements of an artist's work and, in light of this experience, of this epiphany, it makes you review it, appreciate it and better understand it.

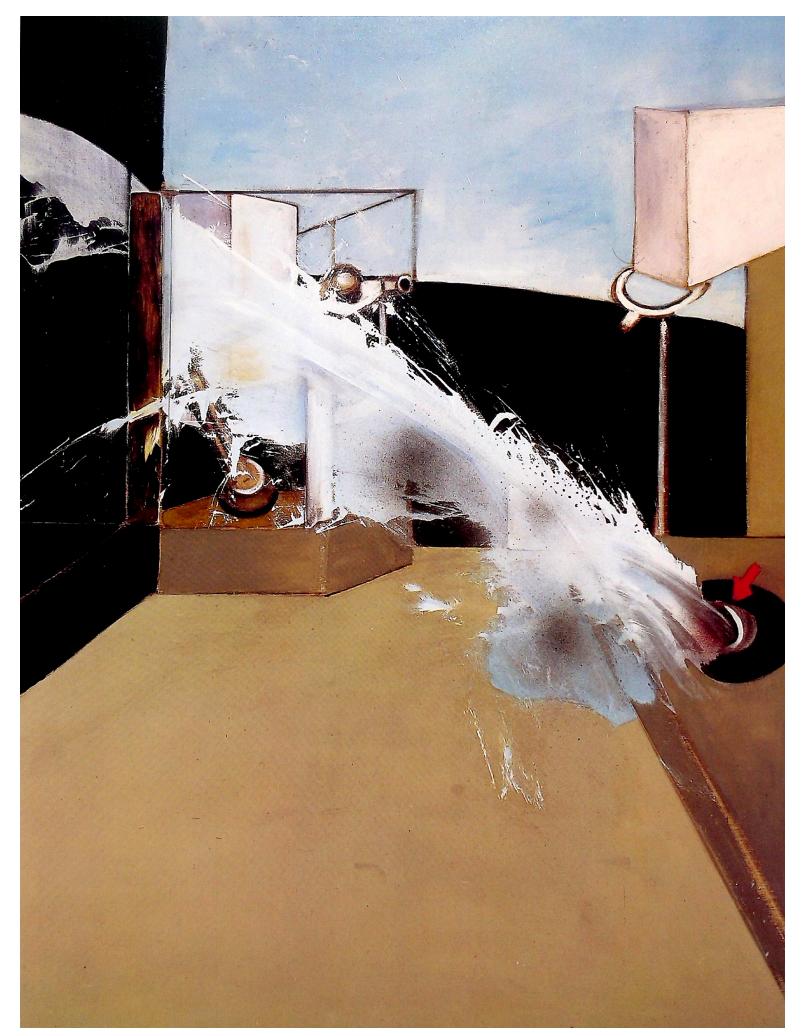

Figure 2. Francis Bacon, Jet of Water, oil and dry transfer lettering on canvas, 1979. 
PGF: It is not just an epiphany about the artist but also about us, because one could not be separated from the other.

VG: Yes, indeed. If I put these elements together, we can say that what seems to happen more easily to me is that these epiphanic moments have some common characteristics, most often they occur with works of contemporary art, more than with works of classical or modern art and are always bound to a strong component of expressive dynamism ... then maybe in 10 years I'll tell you the same thing about Lorenzo Lotto... I don't know... In fact, I visited a very beautiful exhibition on the dream in art in Paris with a small painting by Lotto, "Apollo and the Muses", a very dreamy country scene, with strange colors a little off, a dream atmosphere (Fig. 3). I was there a while looking at it. Of all the paintings that were there, that was the one that really struck me more than the others, even if I couldn't say why.

You visit many exhibitions, and nothing happens. Yes, maybe you see beautiful and interesting things, however this moment so strongly experienced - even on a corporeal level- you don't get it.

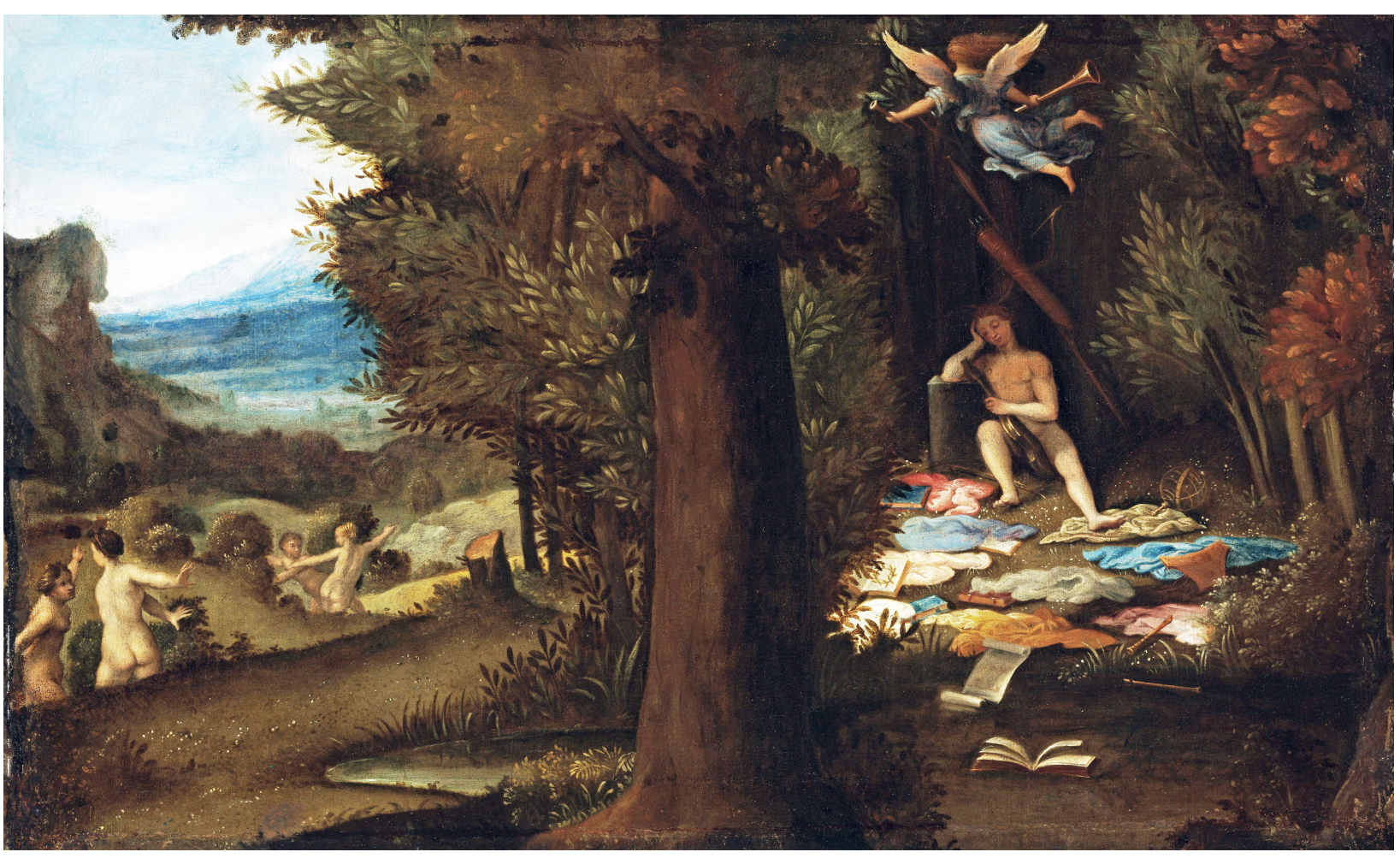

Figure 3. Lorenzo Lotto, Sleeping Apollo and the Muses, oil on canvas (1545 - 49).

PGF: One of the themes of our work is to facilitate this disposition. Epiphany certainly depends on many different factors, we can try to understand how some of these can be amplified, proposed and reproduced. And cognitive neuroscience can certainly be of help in this. 


\section{Conversation with Vittorio Gallese $\quad$ 2:XXXV}

VG: Yes. Epiphany in my opinion is closely connected to what we were talking about before, and that is a more extended temporary dimension, more dilated. In my opinion, it is a mechanism which makes you more receptive, but not only in attentional terms, it also makes you more receptive in the literal sense of the word. It allows the mechanisms which we usually use for the perception of things in the everyday world to be somehow totally, or almost totally, dedicated to that particular perceptual object. So, this concentration summed with a safe distance from the world in which you place yourself - where somehow the potential intrusion of the world causes you to always be a little with your guard up - is put completely or almost completely at the service of the aesthetic experience you are having. This, according to my hypothesis, is one of the elements that somehow makes the aesthetic experience more powerful than the everyday experience of real life.

It is not only thanks to the creativity of the artist that this happens: for example, Cezanne's apple prototypically expresses more "appleness" than a supermarket photograph of apples. This is true not only because Cezanne is a great artist, but also because when you stand in front of Cezanne's apple, you grant yourself this particular aesthetic disposition. When you enter this aesthetic mind-set, you have an enhancement of the embodied simulation mechanisms that I think are somehow one of the load-bearing elements that determine the power of the artistic image with respect to the power of everyday life images.

The traditional way - the usual cognitive way- with which this problem is solved is to affirm that Anna Karenina does not exist. I go to the movies and Scarlett O'Hara is clearly a fictional character, plus she is a moving image on a two-dimensional screen, but I am moved, I participate, I have a set of jolts, I get scared and I get angry, I take a stand, I laugh, I feel terror, and it's all fake. So, what is this? According to classical cognitivism it is the suspension of disbelief. ${ }^{3}$ I just turn a cognitive switch: it's not true, but I pretend that it is.

But this cannot totally explain those situations where the fictional image is more powerful than the real-world image in evoking the same reactions ${ }^{4}$. Because if for the two hours I am watching the film I pretend it is true, I relate to its images as if they were real events, we do not understand the origin of this extra emotion and emotional involvement that we often experience when we immerse ourselves in a fiction storytelling.

PGF: Maybe this is the enhancement you were talking about earlier.

VG: There is something missing, with respect to the translation in cognitive terms of the aesthetic experience beyond the cognitive entry into a specific functional dimension. Unlike what many people say when they talk about the aesthetic experience, namely that they are "quasi-emotions", I find that those evoked by the aesthetic experience are emotions tout court. These are emotions that are often more powerful than those triggered in real life and in my opinion, they take place almost exclusively in the particular contexts in which we have aesthetic experiences. They are

\footnotetext{
${ }^{3}$ Expression introduced by Samuel Taylor Coleridge: "That willing suspension of disbelief which constitutes poetic faith," in Biographia Literaria, 1817.

${ }^{4}$ The locus classicus for this is Hamlet, Act II, scene 2 where Hamlet contrasts the emotion of an actor in a play he has watched to this own lack of similar emotion as the spectator of the real-life death of his father. Of the actor, he says, "What's Hecuba to him, or he to Hecuba, that he should weep for her?" But then Hamlet calls himself "pigeon-liver'd," "John-a dreams" and "a dull and muddy-mettled rascal" for his lack of emotional reaction to the loss of his father.
}

Studies in Digital Heritage, Vol. 2, No. 1, Publication date: December 2018 
contextualized experiences. When I read a novel, I am in a protected environment, in an armchair or in a bed, I see a film at the cinema or I am again on the sofa in my house, I see a picture and I am in a museum. I am in dedicated spaces: first, low potentially threatening intrusiveness. Second, it is I who decides, I make the choice to immerse myself in this world of fiction. Then, the third element, equally if not even more important, I am still, immobile. This immobility, somehow -it is a hypothesis to be verified and I still don't know how- enhances the simulation mechanisms. If you think about it, the most vivid experiences we make, in addition to the experiences we have in everyday life during waking, are dream experiences. During REM sleep apart from eye movements, our body experiences the lowest level of muscle tone within the circadian cycle of the day. Of course, I do not claim that the aesthetic experience is like a dream. But is it a pure coincidence that such a vivid dream experience takes place parallel to a level of total immobility, to which is added a muscular atony? I don't think so. If I imagine something standing still, I can probably put more resources of my sensorimotor brain into the service of this imaginative activity, than when I imagine the same thing walking. Because if I'm walking, my motor system does two things: it makes me walk and at the same time maybe makes me imagine going up stairs or dancing with someone. Therefore, all these situations that contextualize the aesthetic experience contribute to make it such, that is to give that quotient of otherness with respect to everyday life. But someone may very well shut me up saying: oh well but I listen to audio books while jogging ... but we're talking about something else.

Many years ago at the prose season in Parma which at the time was still all at the Teatro Regio, I arrived in the theatre, I was about to sit in my seat in the stalls, and next to me I found a lady all tied to the chair with ropes: it was Judith Malina, protagonist of a show of the Living Theater. On occasions like this the safety distance is cancelled. The viewer experiences a sense of disturbing disorientation that is voluntarily sought by the artist, but we are talking about another thing. I am talking about a more traditional mechanism, which is manifested when we find ourselves in front of a painting, a sculpture, when we are in the cinema, or when we read a novel. In my opinion, all these elements make the aesthetic experience of a work of art, of a work of symbolic human creativity, special. It is, therefore, not only a cognitive operation, but there is an enhancement of the resources that we normally bring into our daily life, and which are amplified during the aesthetic experience for all these circumstantial factors that give a particular space-time contextualization to my perceptual experience, combined with immobility. This is what I call "liberated embodied simulation," that is, simulation freed from the constraints of the potential intrusiveness of the real world.

PGF: So, we could favor this enhancement of human abilities by creating a sort of direction of the path towards the work?

VG: Yes, yes. I would call it "montage". Eisenstein is an author who in recent years whose films I have frequently watched [Gallese and Guerra 2019] - and the more you spend time with him, the more you discover his absolute genius - analysing his own interpretation, so the editing becomes a bit what you call directing. Yes, it becomes a particular editing of the aesthetic experience.

PGF: Editing the aesthetic experience or directing perceptions ... thinking not only of the amplification of the work of art performed through a "montage" that is expressed in continuity with its contents and its formal qualities; but also a direction of the psychophysical and sensorimotor perception of the user, to free his embodied simulation. 


\section{Conversation with Vittorio Gallese $\quad$ 2:XXXVII}

The exhibition should, therefore, envisage a progressive physical and perceptual approach to the work of art with a modulation of the visitor's emotions. With the path of approach to the work that is articulated as if it were an emanation of it, which shapes the space, the lighting ... developing a narration that gradually leads to the most profound contact with the work of art.

VG: These are all factors that seem to be in the background, but which in reality are not a side dish. Because there is one thing that our brain constantly does, and that is to integrate. The brain in turn realizes a montage, constantly. Then the vision is never purely visible, it is never just an optical solicitation, but also a tactile, sensorimotor, affective solicitation ... and you can say it of all the senses. That is, I see with my skin, I see with my muscles, I see with emotions, as I feel with sight, with touch, with movement, and so on. This is not a hypothesis, there is very solid empirical evidence. There is practically no single modality in the brain. For example, the so-called visual areas can also be activated by tactile stimuli, and vice versa. In our experiment on tactile empathy [Freedberg and Gallese, 2007], when the experimenters touched the hand of participants who kept their eyes closed, in addition to seeing the areas that mark tactile sensations activated, we also saw a particular visual area that is sensitive to the vision of movement being activated: the MT area. The participants, on the one hand, saw absolutely nothing, but on the other, somehow "saw" the movement of the hand that caressed their hand.

PGF: This is consistent with experiments undertaken on the imagination, where one also follows with the eye movement an image that is simply projected into the mind [Farah 1989; Farah et al. 1988; Kosslyn 1981; Kosslyn et al. 2001; Kosslyn et al 2001, Ruggieri 1997].

VG: Yes, therefore, given that the aesthetic experience is always a very particular alchemy, so that work X gives me a certain kind of response and another one to you. It produces different answers at different times even to myself. However, I believe that there is a fairly constant factor in making a work of art particularly appreciable: it is the ability of the artist - not programmatic, indeed most often probably unconscious - to facilitate these integrations. The ability, starting from a single sensory channel, which may be that of the vision, to put in resonance various multimodal or synesthetic integrations.

Neuroscience is not necessary to know these things. If we look at the history of art, at critics, Berenson, for example, spoke of "tactile painters", arguing that Giotto was a more "tactile" artist than Cimabue [Berenson 1896]. Today we come to similar conclusions through another path, but this is what it is.

PGF: This is true, but now you have demonstrated it experimentally, providing evidence that allows the sharing between the different disciplines and favors the transdisciplinary activity in good conscience of the various fields to which they belong.

VG: We give an empirical side to intuitions that in other ways had already been reached many times. From Plato and Aristotle on, as you can see, this interpretative pendulum constantly cancels the body and then exalts it. The same happens with emotions: if we read Giambattista Vico, whom I often compare to the contemporary cognitive linguist George Lakoff, we understand that he was already saying the same things in the eighteenth century. Vico clearly belongs to another historical context, using a different language, but the meaning basically remains that. 
PGF: In fact, I think that the characters that met in Florence during the Renaissance such as Pico della Mirandola, Marsilio Ficino, Michelangelo, Raphael, Leonardo, Benozzo Gozzoli ... were aware of the dynamics we are talking about and knew these same topics by treating them with another language. The Medici had created a multidisciplinary cultural environment based on the centrality of the human being that expressed itself in a multimedia way. Perspective was invented at that time and that context contributed to forming the foundations of Western culture. Earlier as you spoke, I visualized how the epiphany was evoked in the first chapel granted by the Pope to be in a private residence, that of Cosimo dei Medici, who was born in this cultural climate. In the largest hall destined for the churchgoer - today the public - the temporal dimension is experimenced by following the ride of the Magi that goes from left to right clockwise and induces a rotation of the body in that direction with the relative psychophysiological experience. If we want to see the whole fresco, we are forced to assume a movement that parallels the sun's apparent path through the sky and allows us to enjoy the whole ride of the Magi, where the various human events are represented, with causes and effects, going from youth until maturity.

When we arrive in the sacellum, the smaller room with the altar, there is no longer a linear narrative, we are in the presence of angelic choirs and synchrony. You leave a timeline and find another dimension. We are freed from the finite realm of space-time - as you mentioned earlier, this is a prerequisite of an epiphany - and we are in a synchronic dimension. Therefore, we can open ourselves to an epiphany and, symbolically, to the appearance of the Divine, which then becomes the Divine in us.

VG: Yes, we reach the epiphany through a corporeal path.

PGF: A methodological question arises. We need a method that allows a shared approach to the relationship between the individual and the work of art, in order to design and experiment with a path towards epiphany.

VG: This is a subject that can be tackled from many points of view. Using this idea of ??editing as a guiding thread, while we were talking, I was thinking that usually when we go to a museum or see a fresco in a church we start from an overview and then we focus on the details. Within the overall vision you go on to single out different moments, different scenes, you start from the whole and then in some way you metaphorically zoom in on the details. This is a first element. A second element is that we know that when we confront an image, we do not always explore it in the same way, but we explore it in a different way according to the reason for our exploration.

The first experiments of Yarbus, recording the eye movements of people looking at the painting "The Unexpected Guest," showed how people explore this picture and concentrate in different ways and on different points, according to the question that they are asked before looking at it [Martinez-Conde and Macknik 2015].

We also know that, for example, if I am an experienced radiologist, I look at an X-ray in a very different way from a first-year medical student. So, it occurred to me that if we sample the way in which a picture is explored by an expert, and based on this sampling we construct a narrative of the picture that precedes its presentation, zooming in on the details that are looked at by the artist, by the art historian, from the restorer, in some way we prepare vicariously the "generalist" spectator or the 
naive eye to see that ensemble by taking a different visual path, exploiting skills that the common spectator does not have.

If this pictorial narrative precedes the viewing of the whole picture, then it makes you look at it in a different way. I do not say that one must necessarily go and see the details, the details that have been explored by the deconstruction that preceded the holistic experience, but it would also be interesting to see if and how different deconstructions influence the subsequent holistic experience. But maybe I'm talking about things you've already done.

PGF: I tried it in the work for the Chapel of the Magi [Perla Gianni Falvo 2015], where the contact with the artwork was preceded by the experience in the Laboratorio di Lorenzo, a pre-visit room equipped with a multimedia apparatus that allowed us to do what you described: present the overall vision of the fresco while giving the visitor the opportunity to choose, through a natural interaction, details to be enlarged to obtain images that went beyond the capacity of the human eye to perceive reality. The people who had seen the details (with the heads of the subjects enlarged up to 2 meters) later, while they viewed the original, were able to appreciate the details of the brushstrokes in the curls of the hair or in details of the landscape. And this also opened them up to the opportunity to make other personal explorations. During the Workshops the contact with the work was amplified by the presence of augmented reality in a sort of double vision at different scales. I agree with you; I think that the activation of our imaginative capacity must be trained to plan a path within a work of art. Rediscovering known landmarks helps and allows us to design ways to explore the different levels.

VG: Yes, of course, this kind of application of virtual reality is very useful. It is what allows us to see the whole and details. We have recently explored our involvement with moving images through a series of experiments studying how the shooting of an actor's action recorded with different types of camera movements (zoom, dolly and steadicam) influenced the motor involvement of the spectator, measuring the activation intensity of the mirroring mechanism. Steadicam was the most effective technique for evoking an empathic motor resonance in viewers [Heimann et al. 2014]. However, we faced a basic ambiguity, because there were potentially two simulations that could be joined: 1) the simulation of the action being shot; 2) the simulation of camera movement.

In a second experiment we shot an empty scene - where there are no actions to simulate - with a still camera, with the zoom and with the Steadicam. In this second experiment, too, the Steadicam was the winner. In this case, the spectator simulates the movement of the camera's eye [Heimann et al. 2019]. So, even the way I explore an artwork is not necessarily equivalent, if I measure it in terms of the observer's involvement. Shooting techniques have a different quotient of evocation of the embodied simulation mechanisms. We can confidently state this because we have the empirical data.

PGF: These data should guide the design of multimedia content by indicating the most effective shooting techniques. So, for example, the circular movement that I would spontaneously do with Il Tondo Doni or with the Madonna del Cardellino by Raphael I would not do with a Mondrian evoking another type of path.

VG: Indeed, and artists know this very well. In the book "The Empathic Screen," co-written with Michele Guerra [Gallese and Guerra 2019], we talk about camera movements: one of Michele's favourite authors is Kubrick, and somehow you can read each of his films as an experiment with the 


\section{2:XL P. Gianni Falvo}

expressive modalities of different shooting techniques. For example, Barry Lyndon, for diegetic reasons, is the world of watching and not touching, and it is no coincidence that he uses a lot of zooms. The Shining represents Steadicam. Full Metal Jacket has two expressive modes, and, in fact, the film is divided into two parts: there is the boot camp, the training with the comrades, the discipline, the uniformity. Here, the movements are all at right angles, almost all Dolly. Then there is a crazy break, and from the boot camp we switch to combat in Vietnam. There it is all filmed with a hand-held camera. So, in this film there is a perfect synthesis between the use of a particular technological expressive medium that is put at the service of a different diegetic.

PGF: I found this work exciting, an important lesson to be applied in the amplification of a work of art.

VG: Sure. But wanting to contextualize the aesthetic experience, I think of the great contradiction that I find today in the aesthetic experiences at the museum. The museum is, by definition, the world of looking and not touching, just approaching an artwork activates the proximity sensor, which starts the alarm and blocks you. But today our scopic world is largely different: we are in the era of the touch screen!

In two recent works, one written with Michele Guerra and the other alone [Gallese 2018b], I introduced the idea of the "skin screen". Some say that on the new visual devices such as tablets and smartphones with the prêt-à-porter vision, the screen is not seen unless the machine is off, because it would always be as transparent as the cinema screen. When we watch a movie, we don't see the screen but the characters that move on it. In reality, this is false, because the way in which these devices are used -i.e., the relocation of the aesthetic visual experience thanks to the introduction of mobile devices- makes their screen periodically opaque, since there is no longer any mediation operated by the remote control. The viewer acts directly on the image, and when she/he touches the screen to enlarge the image, shrink it, restart it, pause it, go back, move forward, etc., it is no longer transparent but becomes a screen because it is the object on which an action will be directed. The direction of the scopic experience is carried out by the spectator's hand, acting directly on the image. We have an absolute coincidence between technical platform and images. The image somehow lives below a screen that is transparent, but which periodically becomes opaque, returning to be a screen as soon as it is touched. Everything that has been said about 'haptic vision' by many scholars has been profoundly influenced by Merleau-Ponty's contribution. Going back in the history of thought, we discover that Merlau-Ponty gave a series of lectures at the College de France on Maine de Biran, a philosopher of the Napoleonic age who produced a systematic analysis of touch and vision: the eye that touches by Merleau-Ponty's was surely inspired by Maine de Biran.

I have yet to investigate these issues experimentally, so I can only formulate hypotheses. The relocation of the images onto touch screens produces the summation of two activities: there is, on the one hand, the "tactile vision" of which Berenson spoke and the so-called "tactile colors", of which Rothko speaks, intrinsically linked to the formal quality of images, color, and materiality of the image. There is, on the other hand, also another tactile dimension, a more factual one. It derives from the fact that with mobile devices the images are not only seen, but also constantly and literally touched. When looking at a pictorial work - which normally we can look at but not touch - with the screen skin of the mobile device, it is possible that the subsequent experience, even if only visual, could be 
different in some way: maybe an enriched experience, I don't know, I don't think anybody has yet raised the issue. I, therefore, have something to investigate; it is an open but potentially explorable question. This is what I plan to do with a new series of experiments.

PGF: These are exciting aspects to explore. We can build a work project integrating it into a dedicated museum layout, given that in the aesthetic experience also proxemic aspects are involved.

VG: Yes, this is an element which I have yet to speak to you about. You reminded me of it by introducing the adjective "proxemic". Apart from the observation of the images present in books we read, most of the traditional aesthetic enjoyment of paintings, frescoes and films always takes place at a distance greater than that of the peri-personal space. Proxemics means that in different cultures the tolerated closeness of the interlocutor varies. Latin or more southern societies tolerate a closer distance, while in northern cultures the distance that for us Latins is acceptable is perceived as intrusive. There is a very strong psychological dimension of proxemics: the peripersonal space is a space that we jealously defend against the intrusiveness of others because we consider it an extension in space of our bodily Self and its motor potentialities of relationship with the world. This raises a question: can the fact that our aesthetic experience systematically takes place within this jealously guarded space influence its quality? This issue, too, is one I want to investigate soon.

PGF: We could test the encroachment into the peripersonal space by using different measures of augmented reality, up to an immersive environment constituted by the work of art itself. It would be interesting to test the difference between this and a contact obtained through HMD.

VG: One thing that I find paradoxical in videogames is the interaction when, for example, you shoot at a target that you don't see yourself, and you are always behind that extension that moves in a subjective way, according to where the weapon is directed. You can see a gun, but you can't see your hands. When you are in a VR world through a headset, you are immersed in a reality that moves in synch with the movements of the head or the body, as if you were living in the virtual space. But we do not see ourselves and we are paradoxically absent. The body of the observer is never visible.

PGF: Now let's talk about multimodal interaction and the sociability of vision...

VG: As we said before, in the aesthetic experience a certain affective-emotional state is aroused with the contribution not only of vision but also of touch, of motor simulation, etc. When we try to measure these responses in the spectator's body and brain, we expect the same artistic content to determine the same kind of reaction in different viewers. If you measure the heart rate of an audience that is attending a classical music concert, you'd expect that at certain points in the score, everyone responds more or less in unison.

Uri Hasson made a famous experiment comparing a film made by him with "The Good, the Bad and the Ugly" by Sergio Leone [Hasson et al. 2008]. Sergio Leone's film causes the brains of all the spectators to activate in the same way at the same time at certain moments in the film. While Uri's film could not synchronize anything, everyone saw it a little in his own way. This suggests that the artist with his work succeeds in striking viewers very effectively, making them enter in the same way in resonance with the contents of the work itself. While the bad work, the non-work of art, does not have this ability. 


\section{2:XLII P. Gianni Falvo}

PGF: Indeed, a work of art is capable of resonating many information organized on several coherent levels, which in a very special way cause this type of result. We should implement a sort of reverse engineering of the artwork itself to favor the synchronization you are talking about, but to do so the contribution of different disciplines in the design phase is necessary.

VG: Sure. I would never have started the empirical study of aesthetic experience alone, I mean, without collaborating with scholars who bring along their specific knowledge in the human sciences on these topics. For me this is a starting assumption, a necessary starting condition.

PGF: I know, and I would like to elaborate this point with you. We know how beautiful and fruitful it is to work with experts of different fields in a more transdisciplinary rather than multidisciplinary way. But in the daily work of exhibition design, even with research aspects, it is difficult to do so.

VG: In the book I authored book with Michele Guerra, we wrote that the multidisciplinary approach brings with it a double gain. There are artists like James Turrell and Olafur Eliasson who do their work capitalizing upon their background in optics and psychophysics. Ori Gherst, an artist with whom we collaborated on the tactile quality of images, is also expert in optics and psychophysics. In practice, I try to do a sort of cognitive archaeology. I try to understand where the aesthetic experience comes from, how it materializes, what is the carnal, neurophysiological base that underlies this experience, deconstructing the concepts, the words that are normally used to describe it. These concepts are the result of a historical stratification of the language of art history, aesthetics, psychology, etc. I am interested in understanding its genesis with the tools of neuroscience.

The same tools of neuroscientific investigation can be used by asking very different questions. For example, if we talk about beautiful or sublime, we can try to localize this concept in particular regions of the brain. It's an interesting way to use neuroscience to talk about aesthetics, but it's not mine.

I'm not so interested in locating concepts in the brain. I am interested in understanding the neural and physiological mechanisms from which these concepts are generated. It is a completely different approach. I am interested, for example, in understanding what happens when we talk about Einfühlung. Robert Vischer23 wrote that when we place ourselves in front of the work of art, we explore its formal characteristics, so to speak, from within, feeling precisely what is inside the work. What is this "feeling inside"? How does it work, what are the contributions of brain and body in the genesis of this feeling inside? What is the nature of the Einfühlung when explored from an empirical perspective? However, before starting the experiments I need to know what I am looking for, to have clear definitions, to know questions to clarify by testing a subject, and that I cannot do on my own. You have to study a lot and have at hand the experts about these issues, such as art historians or film scholars. A different path is unthinkable for me. I would never have studied what happens in the brain of whoever looks at a work by Lucio Fontana or Franz Kline, if this research had not been preceded by a long course of preparatory study [Umiltà et al. 2012; Sbriscia-Fioretti et al. 2013]. The theoretical paper I wrote with the art historian David Freedberg was released in 2007, but the first experiment was published five years later-five years of study and preparation [Freedberg and Gallese 2007].

PGF: Understanding the mechanism of the relationship between author / artwork and user is the heart of the matter. It follows that it is necessary to capitalize on the neurosciences for the enhancement of the perceptive capacities lesson of which you spoke before citing your epiphanic 


\section{Conversation with Vittorio Gallese $\quad$ 2:XLIII}

experience. To calibrate from time to time, the various elements that make up the museum exhibits: from the organization of the space to the development of the approach route to the artwork.

VG: No doubt. With David Freedberg, we assumed that net of cultural differences, but also that there were shared effects. The image of a tree has its own intrinsic power that is universal and goes beyond the individual stories or the cultural standards which can be different. A strong point of the studies we have conducted on Fontana's cuts in the canvas is that we have achieved the same results in those who knew Fontana, and even in those who exchanged the artwork for the control stimulus and vice versa, not having the faintest idea who Lucio Fontana was. Those participants never before saw those images, to which they didn't even attribute an artistic status: nevertheless, the effect was there anyway. There are, therefore, very strong effects that are probably little or not at all modulated by individual differences that are biological but above all cultural. These two planes - culture and nature, are almost inseparable. Epigenetics tells us that the relationship with the world changes the expression of genes and even transmits it to subsequent generations. There is a long-standing contrast between natural sciences and cultural sciences. Given the evident different technical skills - this is undoubted - an art scholar and a neuroscientist use different languages and knowledge tools. Both, however, express two complementary aspects of a single, unified human nature.

PGF: One nature. Our work is focused precisely on being human and its relationship with the art that describes its essence. So, the sciences of nature and culture are indispensable together for studying the phenomenon of aesthetic perception.

VG: In fact, for more than two millennia we keep on reformulating the same old questions.

PGF: Maybe we should reverse the point of view. Human life manifests itself in very different ways that continue to declinate in a specific way, however the root is unique. So, if we tune or harmonize the individual viewer of art with his common roots in humanity represented in various ways by an artwork, we succeed in igniting the spark of humanity that unites us. We get the effect of relating a human being to art. Certainly this does not mean everybody having the same experience: everyone will give this resonance its own individual shape.

VG: Yes, of course. One of the strengths of what we now call art is precisely to make the invisible visible. The invisible that the work of art makes visible to me is something that in the end becomes visible, but I never saw it until I perceived that "artistic" image that resonates in me. This occurs very possibly because in everyday life those expressions escape me and are submerged by a background noise that somehow does not allow them to emerge as a signal. In the aesthetic experience there is a frame effect that comes from the focus and contextualization of the experience, then there is another element, probably even more decisive, which is the ability to synthesize and unveil the world of the artist. From the way it makes you see a hand clinging to the arm of a chair, that hand is not just a hand holding the arm of a chair but behind that hand there is a psychological world, there is a world of actions and relationships. A humanity that I discover by looking at one hand and that tunes me, focuses me on a detail that maybe might escape me in everyday life and that, instead, from that moment can become prototypical and something I bring back to real life by means of the aesthetic experience it triggers. This is one of the aspects that makes art so fundamental. Art is the representation and/or transfiguration of reality in a process of externalization. This is the other aspect that has always fascinated me. That is, it is true that everything is produced by the body and 


\section{2:XLIV P. Gianni Falvo}

the body not only serves to produce the image but is a crucial element of its reception: this suggests to us the logic of the simulation mirroring mechanism. The body is not only the instrument of image production but also a fundamental instrument of its reception through these multimodal resonances of which we spoke earlier. But beyond this aspect, the concept of outsourcing is a common denominator. Our intelligence, our psyche, soul, call it what you wish, is embodied, but struggles to externalize its contents.

Symbolic outsourcing somehow allows us to relocate ourselves in something that prescinds the finite body nature, projecting us into a time dimension very different from that of our biological nature. Art is the product of the body, but we are obsessed with getting out of the body, leaving permanent traces that transcend and survive it.

It is curious to note that ritualization is healthy to the extent that it is shared, whereas when it is solipsistic, it becomes an obsessive-compulsive disorder, it becomes pathology. The different attitude towards ritual behavior when solipsistic compared to when it is collective, constitutes a sort of gauge on the evolutionary path of the species homo sapiens. The collective ritual is the glue that feeds the social and collective identity of a group of humans. Think of ritual dances, initiation rites, mass. Synchronization is probably one of the engines that builds the shared social identity of the group. Somehow, social cohesion and unity create strength.

PGF: And, therefore, it is always the system of relationships that assumes pre-eminence. The relationship with others and with the outside world and art becomes its witness.

VG: Ritualization helps to increase cohesion, while when it becomes a solipsistic gesture it is alienating, not only with respect to one's mental sanity, but specifically because it detaches you from the group.

PGF: These are complex phenomena, and there is no alternative but to study them. We need to put together experts in the humanistic and scientific disciplines to address the issues from various perspectives.

VG: This is what I would like to do, but I find it difficult to do so, because ours is the country of scientific-disciplinary sectors, there is a fragmentation of knowledge and it is more difficult for us to do this kind of things.

PGF: I agree, I often see presentations by various specialists being juxtaposed instead of integrated. The curator proposes an exhibition, so an installer is called in. Then they add someone who plays the multimedia part and someone who takes care of the music, and so on. Most of the time this happens in a linear sequence rather than a synchronic way. There are all the raw human "ingredients", but it should be planned, and the various experts should truly collaborate in real time right from the start...

VG: Ab origine!

PGF: Yes, exhibitions should be planned in a coherent way, in order to develop together the project at the service of the user, on the one hand, and the amplification of the artistic subject, on the other.

VG: When you put yourself into a multidisciplinary perspective you have to work harder, because you need to understand each other's termini tecnici. And how do you understand each other's language? You have to study it. If you are German and I am English, if we both decide to speak Italian, we must

Studies in Digital Heritage, Vol. 2, No. 1, Publication date: December 2018 
both study Italian. But if we want to understand each other by continuing to speak our respective languages, German and English, I must know a minimum of German, as you must know a little English, otherwise it is really a dialogue between deaf. If you tell me, "ach, there is nothing but German," and I say, "ah, there is only English", in the end everyone goes their own way, possibly after having insulted each other! It can't work that way. There are various reasons, in increasing order of triviality, that make the multidisciplinary approach difficult: one of the main obstacles is represented by the fact that it costs time and effort to learn the history, language and problems of disciplines unfamiliar to us. So, if you are an established figure in your field, it may also be legitimate to ask, I, an art historian, an expert in aesthetics, an anthropologist, but what need do I have to study the brain? Or, let's take the neuroscientist who decides to study the visual system by applying it to pointillism: he can study why from an optical-physiological point of view Seurat should be seen at a certain distance. To do this, perhaps there is no need to know everything about the evolution that led to pointillism. But the story changes if your ambitions are not just about psychophysics, and involve aspects that raise really important questions, such as what aesthetic experience is, why we like art and why we do it, what is the way to optimize its experience, everything we talked about so far. If you want to enter into this multi- or trans-disciplinary perspective, you have to spend the night studying, in short, you have to do your "homework". So, this is the most logical and noble justification, up to the pettiest argument of the defense of one's own "disciplinary silo", the disciplinary autonomy of which feels threatened. There are people who unfortunately still think this way...

PGF: Yes, and above all we lose sight of the true objective of our work: which is to understand what happens when we are in front of an artwork, that speaks to us of our humanity and why the aesthetic experience is more powerful than the everyday experience.

VG: My research leads me to emphasize not only the performativity of the realization of the work, but also that of its experience. Through the simulation of the gesture that led to its creation, you change the way you educate people about the aesthetic experience.

PGF: Paraphrasing you: to experience in a complete way the humanity of art through an embodied simulation.

VG: I don't want to absolutize it, of course there is much more to the aesthetic experience, for example the more purely linguistic-cognitive aspects. But the bodily level of aesthetic enjoyment cannot be ignored. Years ago, at the MART in Rovereto I had a debate with an Italian philosopher who claimed that all the mechanisms I have described up until now say nothing about the true meaning of the work of art. As if there were only one meaning... it seems absurd to me to limit aesthetic experience to the meaning of art.

In another debate I had in London, an analytical philosopher said that the experience of a film consists in deriving from the images the propositional attitudes (intentions and beliefs) that the director wants to arouse in the audience. According to this perspective, film experience would amount to an abstruse metacognitive operation, without any role left for the body. All this looks to me as implausible cognitive determinism

In these first ten years, mine was a minimalist project, which essentially consisted in this: to demonstrate that if we want to talk about aesthetic experience we cannot stop at a metarepresentational linguistic-cognitive level. There's more. The next step is to understand how much 
we can move forward using this embodied account of aesthetics, also addressing aspects that up until now have been separated from empathic involvement: I speak of explicit aesthetic judgment. The subject of objective aesthetic judgment has always been eminently interpreted according to the terms dictated by the cultural and cognitive canon, keeping passions at bay, that is, silencing the components we have studied so far. So now, the second step is to understand also how this objective aesthetic is instead - in some way - attributable to the mechanisms of empathic relationship with the work.

Is the aesthetic judgment of beauty really all circumscribed to the cognitive-linguistic superstructure, the only one supposedly allowing us to give a detached aesthetic evaluation? Or is aesthetic judgment also indeed dependent on bodily sensitivity? This is an empirical question we are now trying to address. The first results seem to indicate - contra Kant - that even when one is called upon to evaluate the objective beauty of a work, in doing so one uses the bodily resonance that the work is capable of arousing in the beholder. We have recently demonstrated how the aesthetic evaluation of Renaissance pictorial works that portray physical pain expressed by the face is significantly increased when the observer assumes a mimic attitude of the face congruent with the expression of pain portrayed in the painting. When the objective beauty of paintings depicting faces with neutral expression must be assessed, this manipulation is, however, ineffective.

It is only the beginning, but these results are encouraging and should lead us to a certain caution in taking for granted aesthetic concepts that we have accepted uncritically till now. This also is an interesting contribution that neuroscience can offer to the aesthetic debate.

PGF: Thank you for your time.

\section{REFERENCES}

Bernard Berenson. 1896. The Florentine Painters of the Renaissance. G. P. Putnam's Sons. New York. Retrieved on December 282018 from https://archive.org/details/florentinepaint01beregoog

Martha J. Farah. 1989. Mechanisms of Imagery-Perception Interaction. Journal of Experimental Psychology: Human Perception and Performance (15) 203-211.

Martha J. Farah, Katherine M. Hammond, David N. Levine, Ronald Calvanio. 1988. Visual and Spatial Mental Imagery: Dissociable Systems of Representation. Cognitive Psychology (20) 439-462.

David A. Freedberg and Vittorio Gallese. 2007. Motion, emotion and empathy in esthetic experience. Trends in Cognitive Sciences, 11: 197-203.

Vittorio Gallese. 2018a. Empatia ed Esperienza Estetica. Una Prospettiva Neuroscientifica. Conference in the series Stati D'Animo - Arte e Psiche tra Previati e Boccioni, Palazzo dei Diamanti, Ferrara (Italy), April 5, 2018. Retrieved December 20, 2018 from https://youtu.be/yOZeAWtQh6k.

Vittorio Gallese. 2018b. Naturalizing aesthetic experience. The role of (liberated) embodied simulation. Projections, 12, 2, 50-59. https://doi.org/10.3167/proj.2018.120207

Vittorio Gallese and Michele Guerra. 2019. The Empathic Screen. Cinema and Neuroscience. Oxford University Press, October 2019.

Perla Gianni Falvo. 2015. Augmented Reality in museums: Design and evaluation with cognitive technologies. Workshop with impact assessment in the Chapel of the Magi in Palazzo Medici 


\section{Conversation with Vittorio Gallese $\quad$ 2:XLVII}

Riccardi, Florence. Proceedings of the 19th International Conference on Cultural Heritage and New Technologies 2014 (CHNT 19), Vienna 2014. Retrieved on December 282018 from http://www.chnt.at/wp-content/uploads/eBook_CHNT19_Gianni-Falvo.pdf.

Hasson, U., Landesman, O., Knappmeyer, B., Vallines, I., Rubin, N., \& Heeger, D. 2008.

Neurocinematics: The neuroscience of films. Projections: The Journal for Movies and Mind, 2, 126.

Heimann K., Umiltà M.A., Guerra M., Gallese V. 2014. Moving mirrors: A high density EEG study investigating the effects of camera movements on motor cortex activation during action observation. Journal of Cognitive Neuroscience, 26, 9, 2087-2101. https://doi.org/10.1162/jocn_a_00602

Heimann K., Uithol S., Calbi M., Umiltà M.A., Guerra M., Fingerhut J., Gallese V. 2019. Embodying the camera: an EEG study on the effect of camera movements on film spectators' sensorimotor cortex activation. Plos One, 14, 3, e0211026. https://doi.org/10.1371/journal.pone.0211026

Stephen M. Kosslyn. 1981. The Medium and the Message in Mental Imagery: A Theory. Psychological Review (88) 46-66.

Stephen M. Kosslyn, Giorgio Ganis, William L. Thompson. 2001. Neural Foundations of Imagery. Nature Reviews: Neuroscience (2) 635-642.

Martinez-Conde, S. and Macknik, S. L. 2015. From Exploration to Fixation: An Integrative View of Yarbus's Vision. Perception, 44, 884-899. https://doi.org/10.1177/0301006615594963

Giacomo Rizzolatti, Corrado Sinigaglia. 2006. So quel che fai, il cervello che agisce e i neuroni a specchio, Raffaello Cortina Editore.

Vezio Ruggieri. 1997. L'esperienza estetica. Fondamenti psicofisiologici per un'educazione estetica, Armando editore, pp. 105-111.

Sbriscia-Fioretti B, Berchio C, Freedberg D, Gallese V, Umiltà MA. 2013. ERP modulation during observation of abstract paintings by Franz Kline. PloS ONE 8, 10, e75241. https://doi.org/10.1371/journal.pone.0075241

Umiltà, M.A., Berchio, C., Sestito M., Freedberg, D., and Gallese, V. 2012. Abstract art and cortical motor activation: An EEG study. Frontiers in Human Neuroscience, 6: 311.

https://doi.org/10.3389/fnhum.2012.00311.

Received December 2018.

Studies in Digital Heritage, Vol. 2, No. 1, Publication date: December 2018 